\title{
Experimental Demonstration of Nadir Echo Removal in SAR Using Waveform Diversity and Dual-Focus Postprocessing
}

\author{
Se-Yeon Jeon ${ }^{\circledR}$, Member, IEEE, Thomas Kraus ${ }^{\circledR}$, Ulrich Steinbrecher, Gerhard Krieger ${ }^{(}$, Fellow, IEEE, \\ and Michelangelo Villano ${ }^{\circledR}$, Senior Member, IEEE
}

\begin{abstract}
Synthetic aperture radar (SAR) provides high-resolution images for remote-sensing applications regardless of sunlight and weather conditions. The pulsed operation of SAR may lead to an occurrence of nadir echoes in SAR images that significantly affect the image quality in case the pulse repetition frequency (PRF), which is not properly constrained within the SAR system design. As an alternative, pulse-to-pulse variation of the transmitted waveform and dual-focus postprocessing can be exploited to remove the nadir echo and alleviate the PRF constraints (also in ScanSAR operation). This work provides a demonstration of the latter concept through an experimental acquisition of the TerraSAR-X satellite. The experiment is designed by selecting the scene and the acquisition parameters in order to have the nadir echo appearing in the SAR image. The waveform variation is achieved by alternating up- and down-chirps on transmit. The analysis of the results shows the effectiveness of dual-focus postprocessing for nadir echo suppression.
\end{abstract}

Index Terms-Dual-focus postprocessing, matched filtering, nadir echo, pulse repetition frequency (PRF), synthetic aperture radar (SAR), TerraSAR-X, up- and down-chirps, waveform diversity.

\section{INTRODUCTION}

$\mathbf{S}$ YNTHETIC aperture radar (SAR) is a powerful remote-sensing technology providing high-resolution images of the earth's surface independent of sunlight and weather conditions [1]. A typical SAR system transmits electromagnetic pulses and receives the reflection from the surface to be imaged while mounted on a moving platform.

Because of the pulsed operation of SAR, the echoes from the desired scene return back at the radar simultaneously with the echoes of succeeding pulses backscattered from the nearest range to the radar, i.e., the nadir. The intensity of the nadir echo can be stronger than that of the scene because of the specular reflection and the shorter distance. Therefore, the quality of the SAR image may significantly be affected by the nadir echo even though the radar antenna is designed to limit the energy toward the nadir direction. The nadir echo typically appears in the SAR image as a bright stripe [2].

Manuscript received May 4, 2021; revised June 15, 2021 and July 1, 2021; accepted July 5, 2021. (Corresponding author: Michelangelo Villano.)

The authors are with the German Aerospace Center (DLR), Microwaves and Radar Institute Oberpfaffenhofen, 82234 Wessling, Germany (e-mail: michelangelo.villano@dlr.de).

Color versions of one or more figures in this letter are available at https://doi.org/10.1109/LGRS.2021.3095566.

Digital Object Identifier 10.1109/LGRS.2021.3095566
Conventional spaceborne SAR systems are designed to avoid the nadir interference in the SAR image through a constrained selection of the pulse repetition frequency (PRF) using a timing (or diamond) diagram that visualizes the nadir and transmit interferences for certain values of PRF and ground range [3], [4]. The PRF constraint additionally imposed by the nadir echoes limits the SAR system performance in terms of swath width and ambiguities. Therefore, nadir echo suppression is desired to alleviate the PRF constraint and thus achieve the given requirements without increasing the system complexity or the antenna height.

A novel concept for nadir echo suppression using waveform encoding and dual-focus postprocessing was proposed in [5]-[7]. This technique introduces a pulse-to-pulse variation of the transmitted waveform and an additional on-ground postprocessing with a dedicated matched filter to remove nadir echoes. This concept is also effective in smearing and, to some extent, suppressing strong range ambiguities [7], [8].

This letter presents a validation of the new nadir echo removal concept through a TerraSAR-X experiment. Section II recalls the waveform-encoded SAR concept, Section III describes how the TerraSAR-X experiment has been designed, Section IV presents the processing results together with a performance analysis, and Section V draws some final conclusions.

\section{WAVEFORM-ENCODED SAR CONCEPT}

The ambiguous echoes of consecutive pulses can be selectively defocused within range compression by introducing pulse-to-pulse variation of different orthogonal waveforms on transmit, e.g., alternating up- and down-chirps [9]. Different waveform variation schemes, e.g., cyclically shifted chirps, can be exploited as well [5]. Fig. 1(a) shows the SAR geometry, where the nadir and the swath are colored in blue and red, respectively. The transmitted pulses and the echoes are illustrated in Fig. 1(b). The nadir echo (in blue) returns simultaneously with the swath echo of the previous pulse (in red) because of the shorter distance to nadir. The echo from the nadir can be defocused while focusing the echo from the swath even though they are superimposed because they correspond to different transmitted waveforms. The successfully defocused nadir echo is less visible but still exists in the background degrading the SAR image quality. 


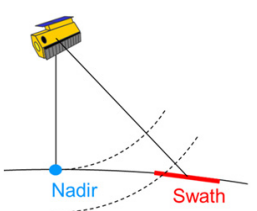

(a)

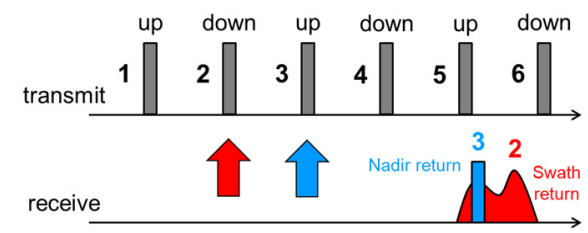

(b)

Fig. 1. (a) Geometry of a typical SAR. (b) Transmitted pulses with waveform variation and corresponding superimposed returns from the nadir and the swath.
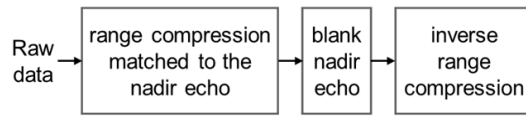

(a)
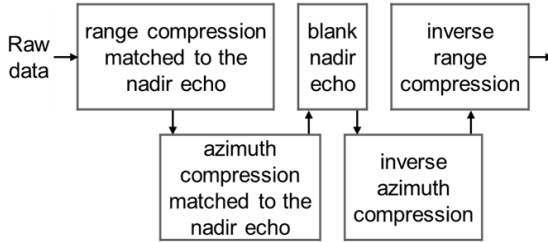

(b)

Fig. 2. Block diagram of the dual-focus postprocessing in (a) rangecompressed domain and (b) focused domain.

Please note that in case the number of traveling pulses between nadir and swath echo is even, the same effect can be obtained by alternating a block of $N$ up-chirps and $N$ down-chirps, e.g., for a number of traveling pulses between nadir and swath echo equal to 2,6 , or 10 , it is enough to alternate two up-chirps and two down-chirps, for a number of traveling pulses equal to 4,12 , or 20 four up-chirps and four down-chirps, and so on and so forth. This consideration further allows extending the technique to ScanSAR operation, where the number $N$ of up-chirps and down-chirps to be alternated might be different for the different bursts, as the number of traveling pulses between nadir and swath echo can be different for the different subswaths.

The defocused nadir echo can be further removed by applying dual-focus postprocessing. The main idea is to focus the raw data using a filter matched to the nadir echo instead of one matched to the useful signal. The nadir echo is then located at a specific range (or a narrow range interval), while the useful signal is defocused over a wide interval of ranges. This offers the opportunity to remove the nadir echo without significant corruption of the useful signal. The location of the nadir echo can be determined either analytically from the PRF, the orbit, and a digital elevation model of the scene, or by an adaptive threshold from the acquired data.

There are two possible ways to perform the blanking of the nadir echos, either in range-compressed data as shown in Fig. 2(a), or in fully focused data as shown in Fig. 2(b). In the latter case, the azimuth compression should be performed considering the range of the nadir echo. Additional steps of azimuth compression and its inverse process are required to blank the nadir echo in focused data, as shown in Fig. 2(b). A further investigation to understand whether

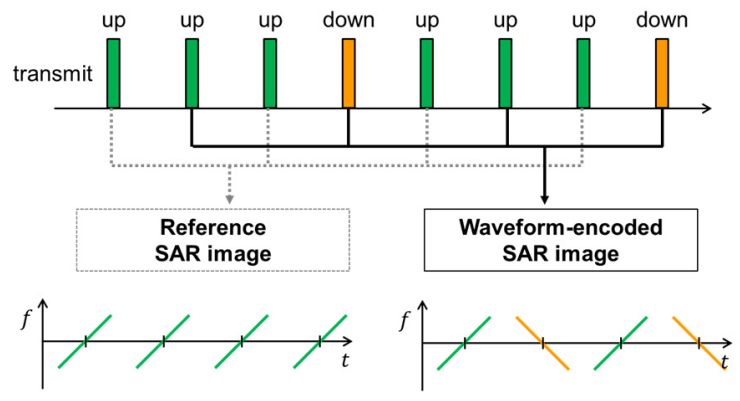

Fig. 3. Waveform variation scheme to obtain interleaved acquisition of a reference SAR image and a waveform-encoded SAR image.

blanking the nadir echo in range-compressed or focused data is preferable and is addressed in Section IV. The nadir echo is simply blanked, i.e., the samples are replaced with some zeros. Another option is to introduce noise with a mean level corresponding to that of the useful signal in order to avoid a possible radiometric scalloping. The data where the nadir echo has been removed are then "inversely" focused back into raw data and focused again using a filter matched to the useful signal. In the final focused image, the information of the scene is negligibly affected, but the nadir echo is significantly suppressed. A detailed point-target simulation can be found in [5].

\section{DESIGN OF THE TERraSAR-X EXPERIMENT}

A TerraSAR-X experiment has been designed to demonstrate the effectiveness of the technique beyond the point-target simulations [10]. In addition to the selection of a proper scene, compared with conventional acquisitions, some timing aspects (e.g., the PRF selection) need to be carefully considered and a pulse-to-pulse variation of the transmitted waveform needs to be introduced.

The scene is selected to maximize the probability of having the nadir echo appearing in the SAR image. In particular, based on the experience with previous TerraSAR-X acquisitions, an area near Tianjin, China, has been chosen, where the signal transmitted to the nadir is backscattered by a calm water surface so that the nadir echo is expected to be strong enough, and the imaged swath includes both natural and artificial scatterers, e.g., buildings.

A waveform variation scheme is devised that allows the interleaved acquisition of a waveform-encoded SAR image with alternation of up- and down-chirps and a reference SAR image with only up-chirps for performance analysis and comparison. Fig. 3 shows the waveform variation scheme, which alternates three up-chirps and a down-chirp, and how the reference and waveform-encoded SAR images are obtained from the acquired data.

The swath to be imaged is chosen to ensure that, using one of the highest available PRFs of the system, the nadir echo of a pulse comes back at the radar together with the scene echo transmitted two pulses in advance (considering the interleaved configuration, i.e., one pulse in advance, if one considers only the reference image or only the waveform-encoded SAR image). The very high PRF, in the order of $6600 \mathrm{~Hz}$ and usually exploited for experimental quad-pol acquisitions, 


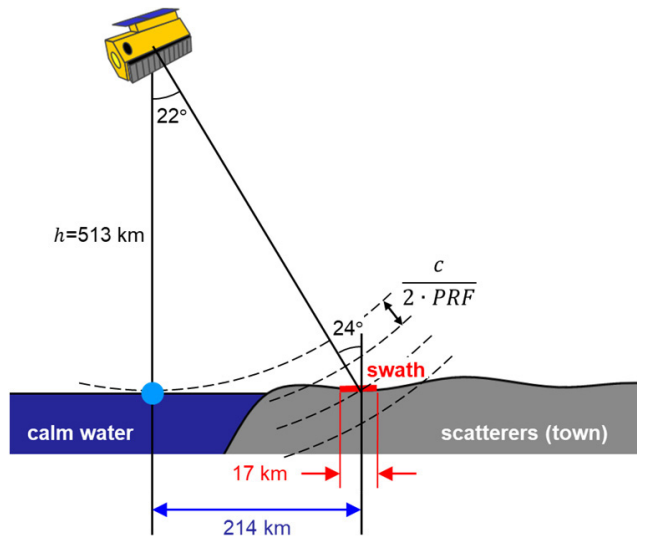

Fig. 4. Geometry of the SAR acquisition for the experiment.

TABLE I

SYSTEM PARAMETERS FOR THE EXPERIMENT

\begin{tabular}{ll}
\hline \hline \multicolumn{1}{c}{ Parameter } & Value \\
\hline Center frequency & $9.65 \mathrm{GHz}$ \\
Bandwidth & $100 \mathrm{MHz}$ \\
Pulse width & $15 \mu \mathrm{s}$ \\
PRF & $6616 \mathrm{~Hz}$ \\
Orbit height & $513 \mathrm{~km}$ \\
Incident angle & $23.7^{\circ}-25.4^{\circ}$ \\
\hline \hline
\end{tabular}

allows unambiguous sampling of each of the two interleaved images, characterized by half the PRF of the acquisition. The imaged ground swath ranges from 207 to $224 \mathrm{~km}$. The nadir echo is expected to appear at a ground range of about $214 \mathrm{~km}$. Furthermore, among the different beams available for the specific swath, the one with the lowest gain difference between the nadir and the point in the scene, where the nadir is expected to appear, is selected. The properties of the selected scene and the geometry of the SAR data acquisition are illustrated in Fig. 4, whereas the system parameters of the TerraSAR-X acquisition are provided in Table I.

The experimental TerraSAR-X acquisition took place in December 2020. A couple of attempts were needed before obtaining data with visible nadir echoes, as moderate wind conditions were enough to reduce significantly the strength of the nadir echoes.

\section{Data Processing and Performance Analysis}

The focused reference image using only up-chirps as in conventional SAR is shown in Fig. 5. Please note that the elevation antenna pattern has not been corrected for and the beam is not symmetrical around the scene center, as it has been chosen to maximize the gain in the nadir echo direction. The nadir echo appearing in the image is indicated with red boxes. The nadir echo is clearly visible in the center of the zoomed-in view image as a bright stripe.

The nadir echo profile averaged across azimuth is shown in Fig. 6. The profile was oversampled to provide a better plot of the peak. The nadir echo occupies 7-8 cells, which

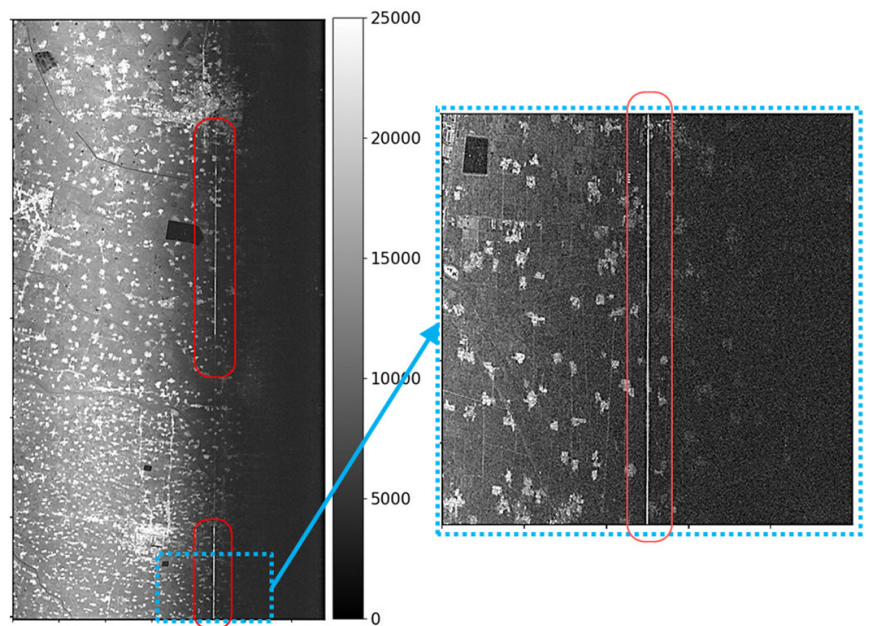

Fig. 5. (Left) Focused reference image with the nadir echo and (Right) zoomed-in view image centered around the nadir echo. Correction for the antenna elevation pattern has not been performed. The horizontal and vertical axes correspond to slant range and azimuth, respectively. The image sizes are $20.6 \mathrm{~km} \times 103.4 \mathrm{~km}$ for the reference image and $7.7 \mathrm{~km} \times 10.7 \mathrm{~km}$ for the zoomed-in view image.

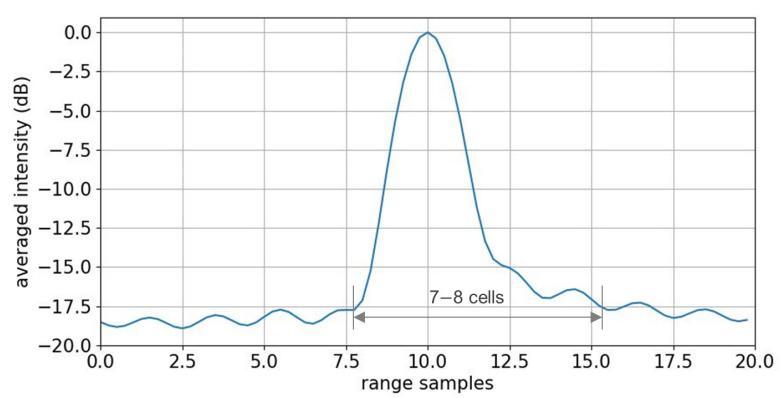

Fig. 6. Zoomed-in view of nadir echo in the averaged range profile.

corresponds to about $70 \mathrm{~ns}$ in fast time or $10.5 \mathrm{~m}$ in slant range.

Fig. 7(a) shows the focused image obtained using up- and down-chirp alternation and a filter matched to the useful signal. The red boxes indicate the area where the nadir echo is located. The waveform-encoded SAR smears the nadir echo in the range direction, as a result of the mismatch between up- and down-chirps. Fig. 7(b) shows the focused image after having removed the nadir echo through dual-focus postprocessing in the range-compressed data.

The postprocessing is not only smearing but significantly removing the nadir echo. The intensity difference images compared with the reference in Fig. 7(c) and (d) show the impact of the waveform encoding and postprocessing on the SAR image. The differences are mainly in the areas where the nadir echo is located, while the other parts of the images remain mostly unaffected. The intermediate steps of the multifocus postprocessing, including blanking of the nadir echo in the range-compressed data, are shown in Fig. 8.

Zoomed-in views of the focused reference, waveformencoded, and postprocessed images are shown in Fig. 9. To better quantify the suppression of the nadir echo avoiding influences on the background, range profiles averaged over 


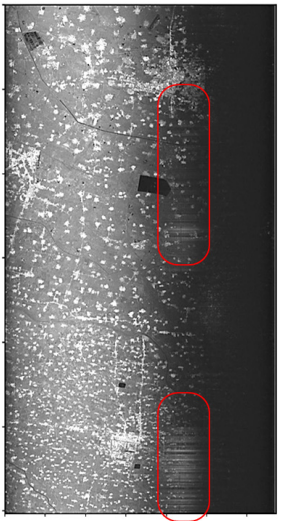

(a)

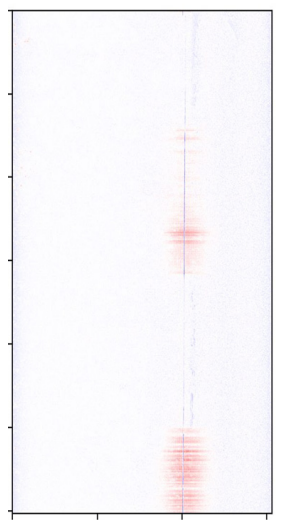

(c)

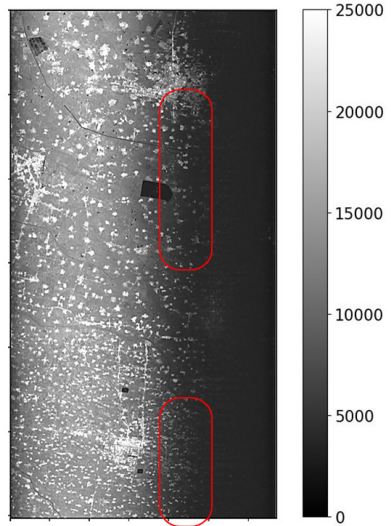

(b)

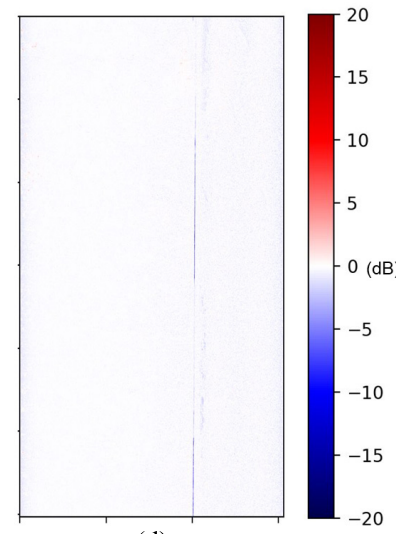

(d)
Fig. 7. Focused image of (a) up- and down-chirp waveform-encoded SAR data and (b) dual-focused postprocessed data. Intensity difference in $\mathrm{dB}$ compared with the reference of (c) up- and down-chirp waveform-encoded SAR data and (d) dual-focused postprocessed data. The horizontal and vertical axes correspond to slant range and azimuth $(20.6 \mathrm{~km} \times 103.4 \mathrm{~km})$, respectively.

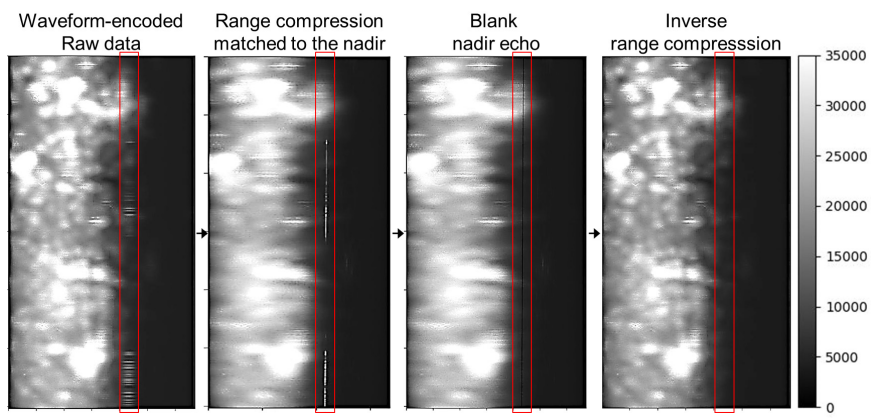

Fig. 8. Intermediate steps of the multifocus postprocessing, including blanking of the nadir echo in range-compressed data. The horizontal and vertical axes correspond to slant range and azimuth $(20.6 \mathrm{~km} \times 103.4 \mathrm{~km})$, respectively.

azimuth are obtained for each of the three images and shown in Fig. 10(a), which refers to the zoomed-in view areas in Fig. 9. The peak of the nadir echo is visible in the range profile of the reference as a blue solid line. The range profile of the waveform-encoded SAR image (green dashed line) shows that the nadir echo is successfully suppressed, but its energy is smeared over the range direction. In the range profile of the postprocessed image (orange dotted line), the nadir echo is

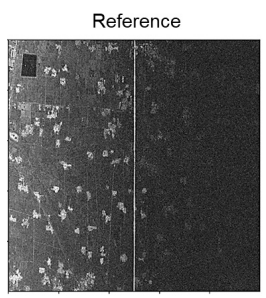

(a)



(b)

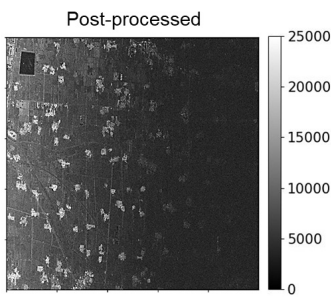

(c)
Fig. 9. Zoomed-in view focused images of the (a) reference, (b) waveformencoded, and (c) dual-focused postprocessed data. The horizontal and vertical axes correspond to slant range and azimuth $(7.7 \mathrm{~km} \times 10.7 \mathrm{~km})$, respectively.

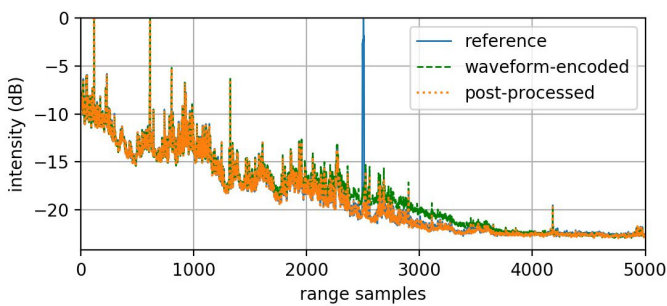

(a)

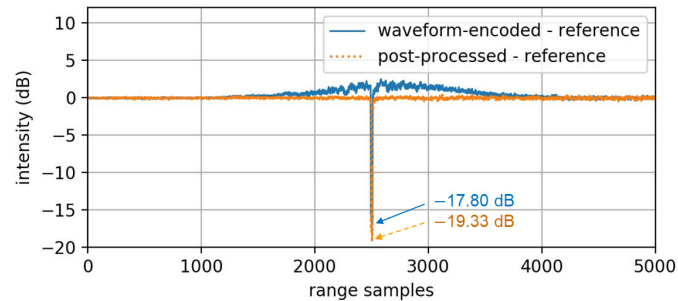

(b)

Fig. 10. (a) Range profiles of the zoomed-in view area averaged over azimuth direction and (b) differences of the averaged range profiles compared with the reference.

significantly suppressed without corrupting the useful signal. The differences of the averaged range profiles compared with the reference are shown in Fig. 10(b). The waveform encoding suppresses the peak of the nadir echo by $17.8 \mathrm{~dB}$ by smearing its energy over the background. The postprocessing suppresses the peak of the nadir echo by $19.3 \mathrm{~dB}$ and has only a negligible effect on the rest of the image. The number of cells to be blanked have been optimized and the final image in Fig. 7(b) has been obtained by blanking 15 cells in the range-compressed data, i.e., the number of cells to be blanked is in the order of magnitude of the nadir echo length.

A further investigation has been conducted to understand whether it is preferable to blank the nadir echo in range-compressed or fully focused data. Both methods suppress the nadir echo successfully without noticeable differences in the final focused image. The averaged range profiles are compared to see the differences between the methods in Fig. 11. The performances may vary depending on the number of blanked cells. If fewer cells are blanked, e.g., seven cells, postprocessing in the fully focused domain suppresses the nadir echo better, while part of the defocused nadir echo is still left if blanking is applied on rangecompressed data, as shown in Fig. 11(a). This could be due to the fact that range cell migration is not corrected 


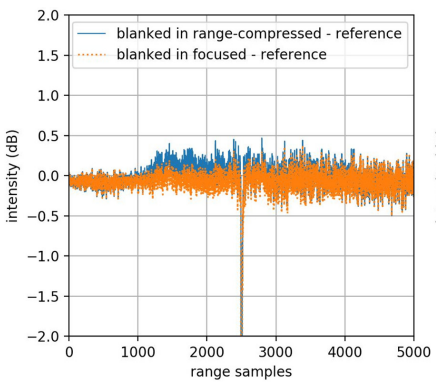

(a)

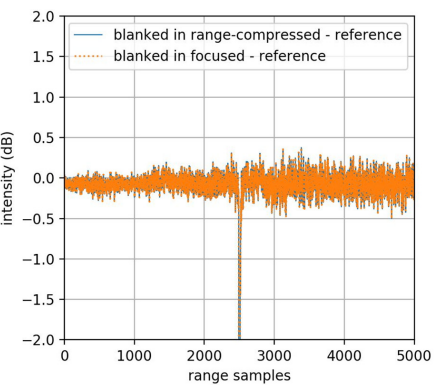

(b)
Fig. 11. Differences of the averaged range profiles that are postprocessed in the range-compressed and fully focused domain compared with the reference for (a) seven blanked cells and (b) 15 blanked cells.

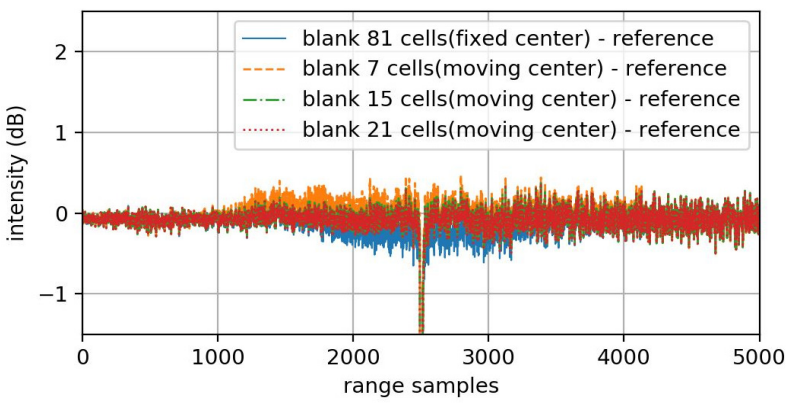

Fig. 12. Differences of the averaged range profiles after postprocessing with blanking in range-compressed data compared with the reference with different blanking options.

for in the range-compressed data. When enough cells are blanked, e.g., 15 cells, the difference between blanking in range-compressed and fully focused data becomes negligible, as shown in Fig. 11(b).

The number of cells to be blanked for the nadir echo removal can be determined depending on the number of cells the nadir echo occupies in the range direction. Note that for a long data take the nadir echo migrates over a number of range cells that may even be larger than its width across range because the distance of the nadir echo changes due to the orbit height variation during the acquisition. The blanking method can be adapted accordingly by either blanking for the whole scene the range interval where at least one nadir echo is located or by moving the blanking window along azimuth. Several blanking methods tested on range-compressed data are compared in Fig. 12. Blanking the full interval of 81 range cells where the whole nadir echo is located (blue solid) is slightly corrupting the useful signal with an energy loss compared with the reference. Blanking seven cells with a moving window (orange dashed) leaves some defocused nadir echo, while blanking 15 or 21 range cells with a moving window (green dashdot and red dotted, respectively) leads to an effective removal of the nadir echo without a visible corruption of the underlying image.

\section{CONCLUSiON}

The innovative waveform-encoded SAR concept using dual-focus postprocessing for nadir echo suppression has been validated with a TerraSAR-X experiment. The TerraSAR-X experiment was designed to have the nadir echo appearing in the SAR image, by selecting the scene to be imaged and the experimental parameters accordingly. A waveform variation based on up- and down-chirp alteration on transmit was used as an example of waveform-encoded SAR. The successful suppression of the nadir echo using the proposed technique was shown through the performance analysis of the TerraSAR-X data. The SAR images obtained using waveform encoding and dual-focus postprocessing were compared with a reference conventional SAR image acquired during the same data take. The waveform-encoded SAR suppressed the nadir echo peak by almost $18 \mathrm{~dB}$ by smearing its energy over the background. The dual-focus postprocessing suppresses the peak of the nadir echo by more than $19 \mathrm{~dB}$ without corrupting the useful signal. The performances obtained for blanking the nadir echo in range-compressed and focused data, and with different window sizes were discussed. This demonstration paves the way to the exploitation of the waveform-encoded SAR concept in current, planned, and future SAR missions, including those exploiting the ScanSAR acquisition mode.

\section{ACKNOWLEDGMENT}

The authors would like to acknowledge their colleagues Dr. Josef Mittermayer and Maxwell Nogueira Peixoto for many constructive discussions and the anonymous reviewers for their helpful comments.

\section{REFERENCES}

[1] A. Moreira, P. Prats-Iraola, M. Younis, G. Krieger, I. Hajnsek, and K. P. Papathanassiou, "A tutorial on synthetic aperture radar," IEEE Geosci. Remote Sens. Mag., vol. 1, no. 1, pp. 6-43, Mar. 2013.

[2] J. Balkoski and F. Bordoni, "Nadir echo properties, a study based on TerraSAR-X data," in Proc. TELFOR, Belgrade, Serbia, Nov. 2012, pp. $420-423$.

[3] J. C. Curlander and R. N. McDonough, Synthetic Aperture Radar: Systems and Signal Processing. New York, NY, USA: Wiley, 1991.

[4] J. Mittermayer, M. Younis, R. Metzig, S. Wollstadt, J. M. Martinez, and A. Meta, "TerraSAR-X system performance characterization and verification," IEEE Trans. Geosci. Remote Sens., vol. 48, no. 2, pp. 660-676, Feb. 2010.

[5] M. Villano, G. Krieger, and A. Moreira, "Nadir echo removal in synthetic aperture radar via waveform diversity and dual-focus postprocessing," IEEE Geosci. Remote Sens. Lett., vol. 15, no. 5, pp. 719-723, May 2018.

[6] M. Villano, G. Krieger, and A. Moreira, "Verfahren und vorrichtung zur rechnergestuetzten verarbeitung von SAR rohdaten," German Patent DE 102017205649 , Dec. 5, 2017.

[7] M. Villano, G. Krieger, and A. Moreira, "Waveform-encoded SAR: A novel concept for nadir echo and range ambiguity suppression," in Proc. 12th Eur. Conf. Synth. Aperture Radar (EUSAR), Aachen, Germany, 2018, pp. 1-6.

[8] L. Dell'Amore, M. Villano, and G. Krieger, "Assessment of image quality of waveform-encoded synthetic aperture radar using real satellite data," in Proc. 20th Int. Radar Symp. (IRS), Jun. 2019, pp. 1-10.

[9] J. Mittermayer and J. M. Martinez, "Analysis of range ambiguity suppression in SAR by up and down chirp modulation for point and distributed targets," in Proc. IEEE Int. Geosci. Remote Sens. Symp. (IGARSS), vol. 6, Jul. 2003, pp. 4077-4079.

[10] S.-Y. Jeon, T. Kraus, U. Steinbrecher, M. Villano, and G. Krieger, "A TerraSAR-X experiment for validation of nadir echo suppression through waveform encoding and dual-focus post-processing," in Proc. Eur. Conf. Synth. Aperture Radar (EUSAR), Leipzig, Germany, 2021, pp. $1-5$. 